\title{
Length-dependent motions of SARS-CoV-2 frameshifting RNA pseudoknot and alternative conformations suggest avenues for frameshifting suppression
}

\section{Shuting Yan}

New York University

Qiyao Zhu

New York University

Swati Jain

New York University

Tamar Schlick ( $\sim$ schlick@nyu.edu )

New York University https://orcid.org/0000-0002-2392-2062

\section{Article}

Keywords:

Posted Date: January 4th, 2022

DOI: https://doi.org/10.21203/rs.3.rs-1160075/v1

License: (c) (i) This work is licensed under a Creative Commons Attribution 4.0 International License. Read Full License

Version of Record: A version of this preprint was published at Nature Communications on July 25th, 2022. See the published version at https://doi.org/10.1038/s41467-022-31353-w. 


\title{
Length-dependent motions of SARS-CoV-2 frameshifting RNA pseudoknot and alternative conformations suggest avenues for frameshifting suppression
}

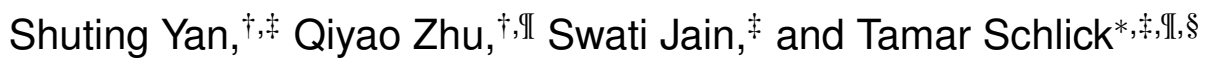 \\ $\dagger$ These authors contributed equally to this work. \\ $\ddagger$ Department of Chemistry, New York University, New York, NY 10003 U.S.A. \\ IICourant Institute of Mathematical Sciences, New York University, New York, NY 10012 U.S.A. \\ $\S N Y U-E C N U$ Center for Computational Chemistry, NYU Shanghai, Shanghai 200062, P.R. China \\ E-mail: schlick@nyu.edu
}

\section{Abstract}

Conserved SARS-CoV-2 RNA regions of critical biological functions define excellent targets for anti-viral therapeutics against Covid-19 variants. One such region is the frameshifting element (FSE), responsible for correct translation of viral polyproteins. Here, we analyze moleculardynamics motions of three FSE conformations, discovered by graph-theory analysis, and associated mutants designed by graph-based inverse folding: two distinct 3-stem H-type pseudoknots and a 3-way junction. We find that the prevalent H-type pseudoknot in literature adopts ring-like conformations, which in combination with $5^{\prime}$ end threading could promote ribosomal pausing. An inherent shape switch from "L" to linear that may help trigger the frameshifting is suppressed in our designed mutant. The alternative conformation trajectories suggest a stable intermediate structure with mixed stem interactions of all three conformations, pointing to a possible transition pathway during ribosomal translation. These observations provide new insights into anti-viral strategies and frameshifting mechanisms.

\section{Introduction}

In less than two years, COVID-19 with its novel infectious agent SARS-CoV-2 has already caused more than 266 million infections and 5 million deaths worldwide. Although the development of multiple vaccines has provided hope for a post-pandemic world, new virus variants with higher infectivity and increased ability to evade the immune system require us to maintain vigilance. Thus, the identification of novel anti-viral therapeutic targets and development of drugs against them remains a priority.

The single stranded SARS-CoV-2 RNA genome of 29,891 nucleotides includes two overlapping, shifted open reading frames ORF1a and $1 b$, which encode for viral polyproteins that begin the viral protein production. To correctly translate both polypeptides, the virus utilizes programmed -1 ribosomal frameshifting ( -1 PRF) to stall and backtrack the ribosome by one nucleotide to bypass the stop codon near the start site of ORF1b.

First discovered in the Rous sarcoma virus in $1985,{ }^{1}$ the 1 PRF stalling of the ribosome is associated with a small $(<100-n t)$ frameshifting element. ${ }^{2}$ SARS-CoV-2 similarly employs such a frameshifting element (FSE) located at the ORF1a/1b junction. This FSE consists of a 7-nt slippery site and a downstream 77-nt stimulatory region, which typically folds into an H-type pseudoknot (Fig. 1). The functional importance and high conservation of the FSE make it a promising candidate for anti-viral drugs and gene therapy; for example, in the latest Omicron variant, there are 31 new mutations in the spike gene region with respect to the previous variants, but no change in the FSE (Fig. S1). ${ }^{3-6}$ Whether frameshifting is orchestrated by the FSE acting as a "road blocker" or through more complex conformational switches remains unknown. ${ }^{7-13}$ Hence, exploring the secondary (2D) and tertiary (3D) structural dynamics of the FSE during translation is essential for both untangling the frameshifting mechanism and developing anti-viral strategies.

Unlike the stem-loop structure for HIV-1 FSE ${ }^{15}$ or the 2stem pseudoknot for IBV FSE, ${ }^{16}$ the assumed structure for SARS-CoV-2 FSE is a 3-stem H-type pseudoknot, where the Stem 1 loop binds the $3^{\prime}$ end to form Stem 2, and Stem 3 lies between them (Fig. 1). This motif has been reported by chemical probing, Cryo-EM, NMR, crystallography, ${ }^{3,17-23}$ and molecular dynamics (MD). ${ }^{24-26}$ The Cryo-EM studies also suggest an "L" shape pseudoknot with coaxial stacking of Stems 1 and 2 which form the pseudoknot, and an extruding Stem 3 in the coaxial plane. ${ }^{20,21}$ In contrast, a recent crystallography study observes a vertical stacking of the 3 stems. ${ }^{23}$ Using our coarse-grained RNA-As-Graphs (RAG) representation as dual graphs, ${ }^{27-30}$ where double-stranded stems are vertices and single-stranded loops are edges, we 

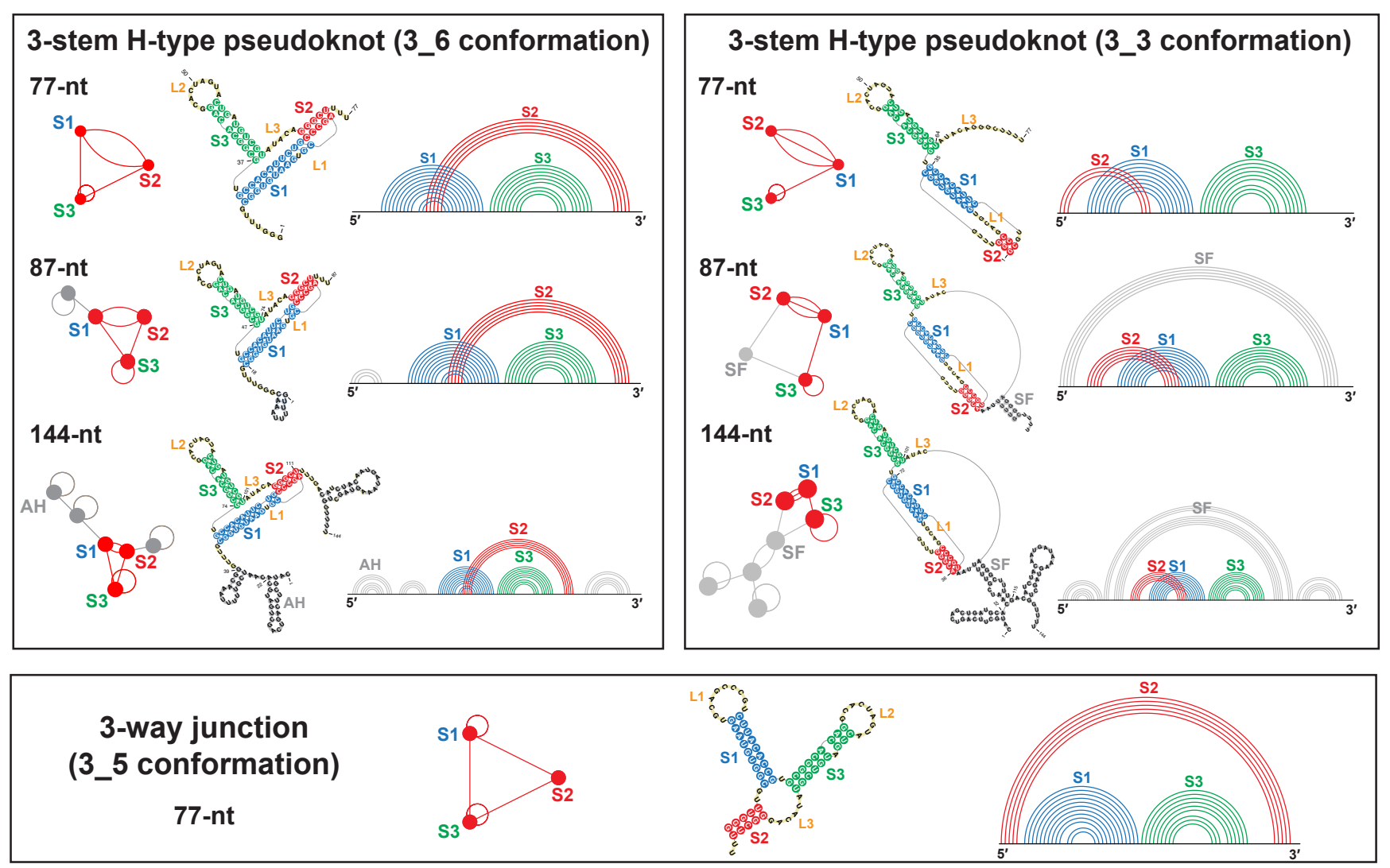

Figure 1: Secondary structures of the three FSE conformations at different lengths we study, along with their arc plots and corresponding dual graphs. For 77-nt, the three conformations 3_6 pseudoknot, 3_3 pseudoknot, and 3_5 junction have common Stems 1 (blue) and 3 (green), while different Stem 2 (red). The two pseudoknots are classified as H-type, ${ }^{14}$ where in $3 \_6$ the loop region of Stem 1 binds with the external single-stranded $3^{\prime}$ end, and in 3_3 the Stem 1 loop binds with the $5^{\prime}$ end. For 87-nt, 10 upstream residues are added that include the 7-nt slippery site, and the 3_3 conformation contains an extra flanking stem SF (grey). For 144-nt, 37 upstream and 30 downstream residues are included, and extra stems (grey) are formed, including attenuator hairpin $\mathrm{AH}$ for 3_6 and SF for 3_3. Stems are represented as vertices in dual graphs, and loops as edges, with the central 3_6, 3_3, and 3_5 submotifs corresponding to the 77-nt FSE region highlighted in red, and the flanking vertices/edges corresponding to the extra stems/loops in grey.

assign this pseudoknot motif as dual graph 3_6 (Fig. 1). ${ }^{3,25}{ }_{92}$ RAG, used to identify key RNA motifs, design novel RNA 93 motifs from building blocks, and perform inverse folding to 94 transform one RNA motif into another, ${ }^{31-36}$ was applied to 95 explore and alter the FSE conformational landscape. ${ }^{3,25} 96$

Indeed, recent works revealed the complexity of the FSE landscape, with alternative conformations including differ- 98 ent pseudoknots ${ }^{3,20,37,38}$ and unknotted structures s,19,20,39-42 $99_{99}$ ( $\mathrm{se}^{3}$ for a detailed comparison). In particular, our prior 100 modeling and SHAPE chemical reactivity experiments re- 101 veal an alternative 3-stem H-type pseudoknot where the 102 Stem 1 loop binds with the $5^{\prime}$ end to form a different Stem ${ }_{103}$ 2 (3_3 dual graph), and a 3-way junction where the $5^{\prime}$ and $3^{\prime}{ }_{104}$ ends pair (3_5 dual graph). ${ }^{3}$ The three conformations (3_6, 105 3_3, and 3_5) have common Stems 1 and 3 (though stem 106 lengths vary) but competing Stem 2 (see Fig. 1). Moreover, our studies have emphasized the high length dependence of 107 the FSE conformations: for short lengths such as 77-nt with- 108 out the slippery site, the 3_6 pseudoknot is the dominant con- 109 formation, and the 3.5 junction is minor; for long lengths 110 such as 87-nt and 144-nt, conformations containing the 3_6 pseudoknot become minor, while those containing the 3_3 pseudoknot become dominant. ${ }^{3}$ As in other positive-sense RNA viruses, ${ }^{43-45}$ structural transitions among these three (and other possible) conformations likely exist and play an important role in frameshifting.

Here we employ several computational 3D structure prediction programs and analyze microsecond MD trajectories of different FSE conformations at three lengths: 77, 87, and 144-nt. We term a particular conformation 3_6, 3_3, or 3_5 according to the central 77-nt FSE fold (Fig. 1). We consider all three conformations for the 77-nt FSE, and 3_6 and 3_3 conformations for 87 and 144-nt FSE. We also study our motif-strengthening mutants that stabilize each conformation over the others.

We identify structural features and motions that help suggest frameshifting mechanisms. For the 3_6 pseudoknot, the $5^{\prime}$ end threading through a ring hole formed by Stem 1 strand and junctions could add mechanical resistance to 
77-nt 36 Pseudoknot

(RNAComposer)

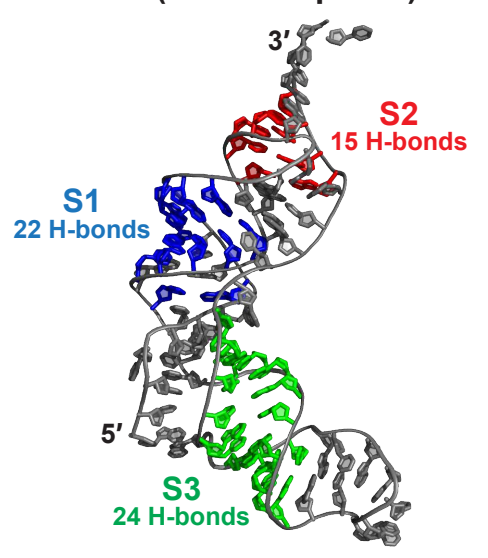

S1, S2 coaxial stacking

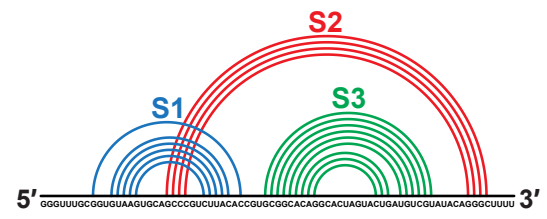

87-nt 33 Pseudoknot (iFoldRNA)

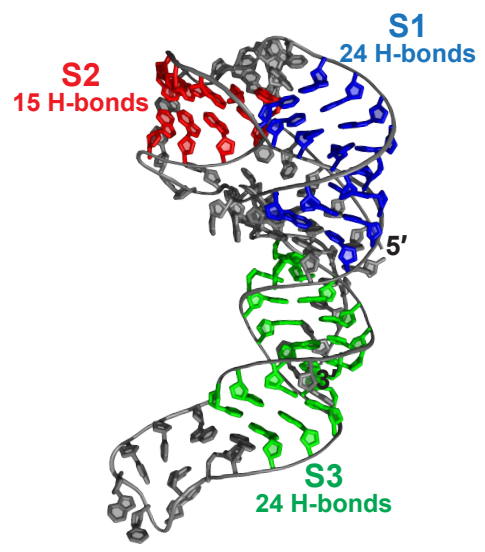

S1, S3 coaxial stacking

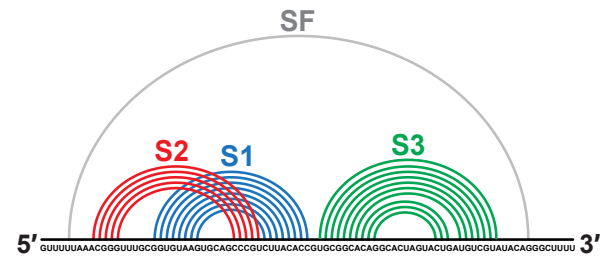

77-nt $3 \quad 5$ Junction (SimRNA)

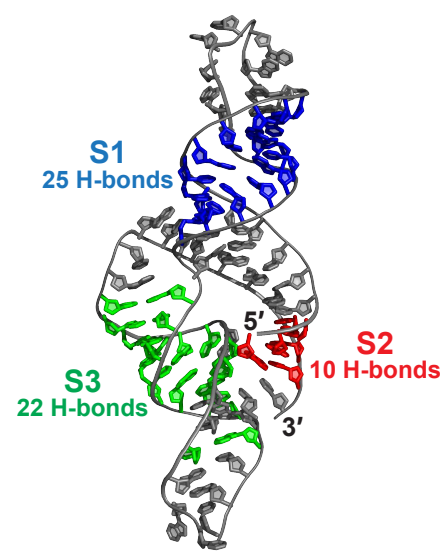

S1, S2 coaxial stacking

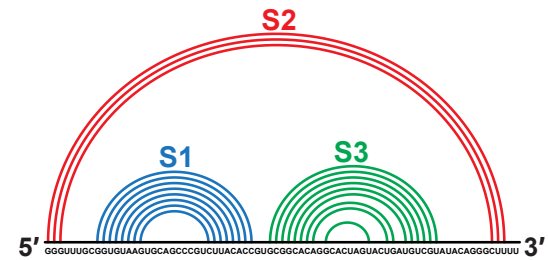

Figure 2: Representative structures for the three FSE conformations. The 77-nt 3_6 pseudoknot (largest MD cluster center structure from RNAComposer) has Stems 1 and 2 coaxially stacked. The 87-nt 3_3 pseudoknot (iFoldRNA) has Stems 1 and 3 coaxially stacked. The 77-nt 3_5 junction (SimRNA) has Stems 1 and 2 coaxially stacked. The 2D structures are extracted using 3DNA-DSSR, ${ }^{46}$ and the numbers of hydrogen bonds formed in the stems (averaged over the last 500 ns of the simulations) are calculated using Gromacs. ${ }^{47}$

ribosomal unwinding and promote longer ribosomal paus- ${ }_{137}$ ing. The axial bending motion of the 3_6 pseudoknot we capture - from an "L" shape (observed by Cryo-EM ${ }^{20,21}$ ) to a more linear shape (observed by crystallography ${ }^{23}$ ) -138 can cause fluctuations in mRNA tension during translation, which might in turn trigger frameshifting. Importantly, our ${ }_{139}$ motif-strengthening mutant suppresses this motion and sta- ${ }_{140}$ bilizes the linear shape.

The large number of different motifs and RNA lengths modeled here for the first time allows us to piece observations and relate them to ribosomal translation. In shorter 3_3 systems, triplet hydrogen bonds that include Stem 2 interactions in all three conformations are present. Such a stable state suggests a potential transition among alternative FSE conformations as different sequence lengths are accessible to the ribosome. Namely, starting from longer sequences, where a flanking stem SF favors 3_3, a transition to 3_6 occurs when the ribosome occludes the slippery site to unwind SF and thus allow formation of alternative Stem 2.

These mechanistic findings hold specific implications for anti-viral strategies. Our work suggests targeting regions that participate in: 3_6 threading ( $3^{\prime}$ helix end of Stem 1), structural switch (Stem $2 / 3$ junction), and pseudoknot stabilizing interactions (hydrogen-bonded triplets near Stem 2). Small molecules or gene editing mutations in these regions could hamper frameshifting.

\section{Results}

\section{Overview}

We model each FSE conformation using several 3D prediction programs (see Methods) and choose representative systems to discuss as follows: RNAComposer models for 3_6 pseudoknot, because they agree well with Cryo-EM experimental structures; ${ }^{20,21}$ iFoldRNA models for 3_3, because they maintain the 3_3 motif for all lengths; SimRNA models for 3_5, because they possess the elongated 3.5 structures seen in most systems. All systems can be found in the Supplementary Information with full descriptions.

Comparing the three representative structures (77-nt for 3_6 and 3_5, and 87-nt for 3_3) in Fig. 2, we note different helical arrangements and stem lengths. The 3_6 pseudoknot has Stems 1 and 2 coaxially stacked, while Stem 3 extrudes from the coaxial plane, forming an "L" shape. The 3_3 pseudoknot has Stems 1 and 3 stacked, and the pseudoknot is more compact. The 3_5 junction has Stems 1 and 2 stacked, but its Stem 2 is near Stem 3 instead of at the top. In all three structures, Stem 2 is much shorter than Stems 1 and 3, and 3.5 has the weakest Stem 2 consisting of mainly G-U wobble base pairs.

Below, we analyze structures and motions obtained from mi- 

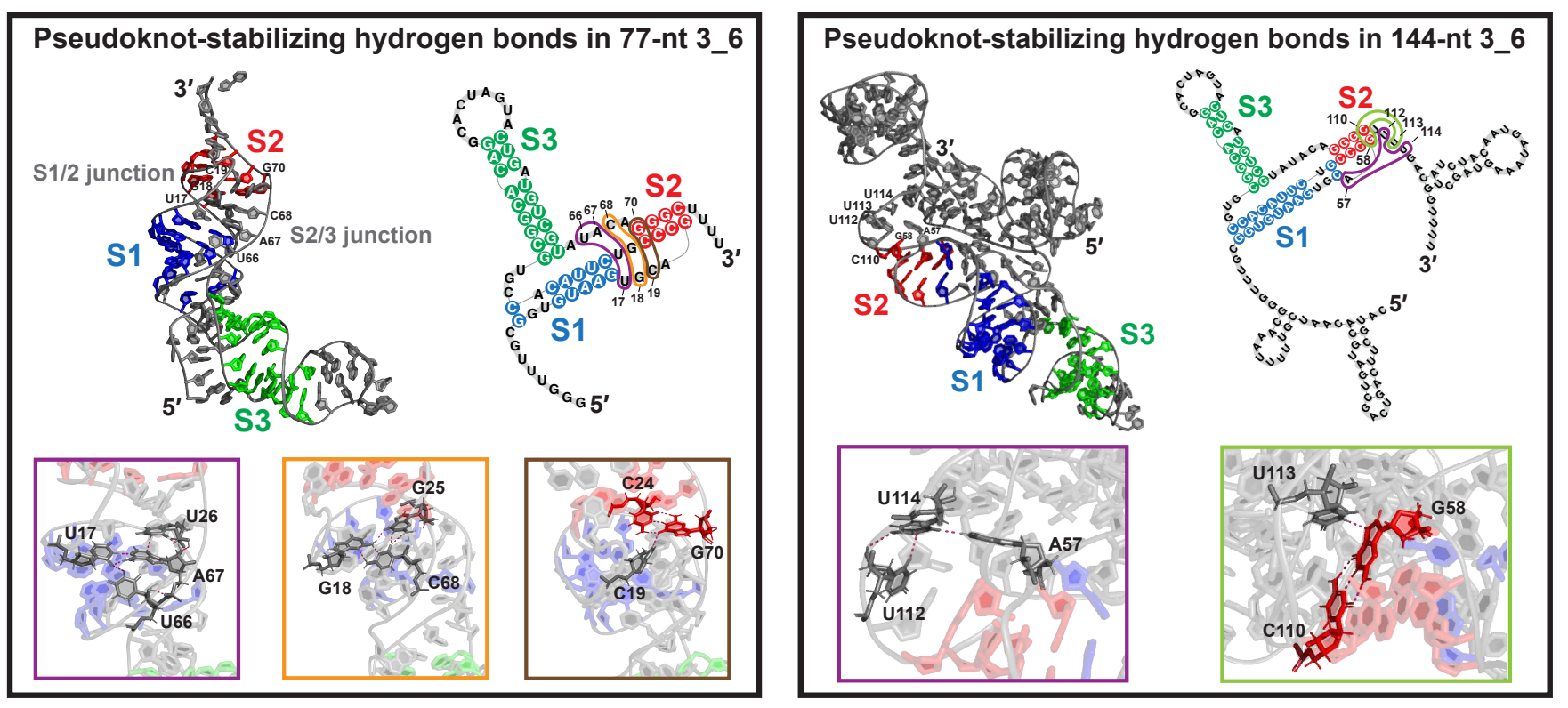

Figure 3: Pseudoknot stabilizing hydrogen bonds in our 3_6 systems. (Left) For 77-nt (RNAComposer), a base quadruplet and two triplets are formed at the Stem 1/2 and 2/3 junctions. (Right) For 144-nt (RNAComposer), two base triplets are formed at the $3^{\prime}$ helix end of Stem 2.

crosecond molecular dynamics simulations for each confor- 191 mation at different lengths. For the 3_6 pseudoknot, we iden- 192 tify critical tertiary interactions, such as hydrogen-bond networks that stabilize the pseudoknot complex (Fig. 3) and 5' 193 end threading that may be associated with ribosomal paus- 194 ing (Fig. 4), and compare our structures with the Cryo-EM 195 models (Fig. 5). ${ }^{20,21}$ For the alternative 3_3 pseudoknot and 196 3_5 junction, we discuss length-dependent flanking stem or ${ }^{197}$ triplet formation (3_3) and the Stem 2/3 interactions (3_5) 198 that provide insights into FSE transitions (Fig. 6). Inherent 199 motions of the three conformations and motif-strengthening 200 mutants are discussed in Fig. 7 and 8. Notably, a key struc- 201 tural switch between the "L" and the linear shape for 3_6 202 that may send frameshifting signals to the ribosome is ab- ${ }^{203}$ sent in the mutant. Global contraction and stretching in the 3_3 pseudoknot, along with triplet interactions, may play a ${ }^{204}$ role in frameshifting structural transitions. The combined ${ }^{205}$ insights suggest target regions for small-molecule binding ${ }^{206}$ and CRISPR gene-editing, as well as a structural transition ${ }^{207}$ pathway (Fig. 9).

\section{Tertiary interactions stabilize the 3_6 pseudo- knot ring-like conformation}

Throughout the microsecond MD simulations, the 3_6 pseu- 215 doknot motif is retained in all systems (Fig. S2). The shorter 216 77-nt structures adopt the "L" shape seen in the Cryo-EM studies ${ }^{20,21}$ (Fig. 3), with smaller radii of gyration (Rg) for ${ }^{217}$ systems more bent (Fig. S3), yet the total RNA potential ${ }^{218}$ energy is about the same for all (Fig. S4). The 87 and 144- ${ }^{219}$ nt structures become more linear, with vertical arrangement ${ }^{220}$ of the three stems (Fig. 3), similar to the recent crystallo- ${ }^{221}$ graphic structure. ${ }^{23}$ The Rg and RNA potential energy in- ${ }^{222}$ crease slightly for 87 -nt, while significantly for 144-nt by $\sim 50 \%$ and $\sim 200 \%$, respectively.

Multiple hydrogen bonds act to stabilize the 3_6 pseudoknot complex (Fig. 3). In the 77-nt "L" shape, unpaired residues in the Stem $1 / 2$ and $2 / 3$ junctions form a quadruplet (U17, U26, U66, A67) and a triplet (G18, G25, C68) that define a short triplex, which is further extended by the binding of junction residue $\mathrm{C} 19$ with the $5^{\prime}$ helix end of Stem 2 (C24-G70). This triplex stabilizes the loose junctions and links the $3^{\prime}$ end tightly near the Stem 1 loop to maintain the pseudoknot. In the 144-nt linear shape, similar triplets are formed by the $3^{\prime}$ helix end of Stem 2 and the downstream loop residues to seal the short Stem 2.

The ring in all 3_6 systems forms by linking the $3^{\prime}$ strand of Stem 1 , Stem $1 / 3$ junction, $5^{\prime}$ helix end of Stem 3 , and Stem $2 / 3$ junction (Fig. 4). In a recent 88 -nt Cryo-EM structure (6.9 $\AA$ resolution), the $5^{\prime}$ end is reported to thread through the ring. ${ }^{20}$ Here, we capture both threaded and non-threaded ring conformations at various sequence lengths (Fig. 4). For 77-nt, the $5^{\prime}$ strand of Stem 1 and the $5^{\prime}$ end can either thread through the ring ("threaded") — possibly hampering ribosomal unwinding and promoting longer ribosomal pausing or wind around the structure ("non-threaded"). For 87-nt, the ring holes are larger, probably due to longer Stem 1, and the extended FSE $5^{\prime}$ end winds around Stem 3 in both threaded and non-threaded structures, though in opposite orientations.

We also identify ring-stabilizing hydrogen-bond networks (Fig. 4). For threaded 77-nt, the Stem 1 loop (U17, U26) and the Stem 2/3 junction (A66, A67) bind to seal the ring top. For threaded 87-nt, a similar triplet (C29, G35, A79) forms at the ring top, and two more at the ring bottom by the $5^{\prime}$ helix end of Stem 1 (C18-G45, G17-U46) with the Stem 

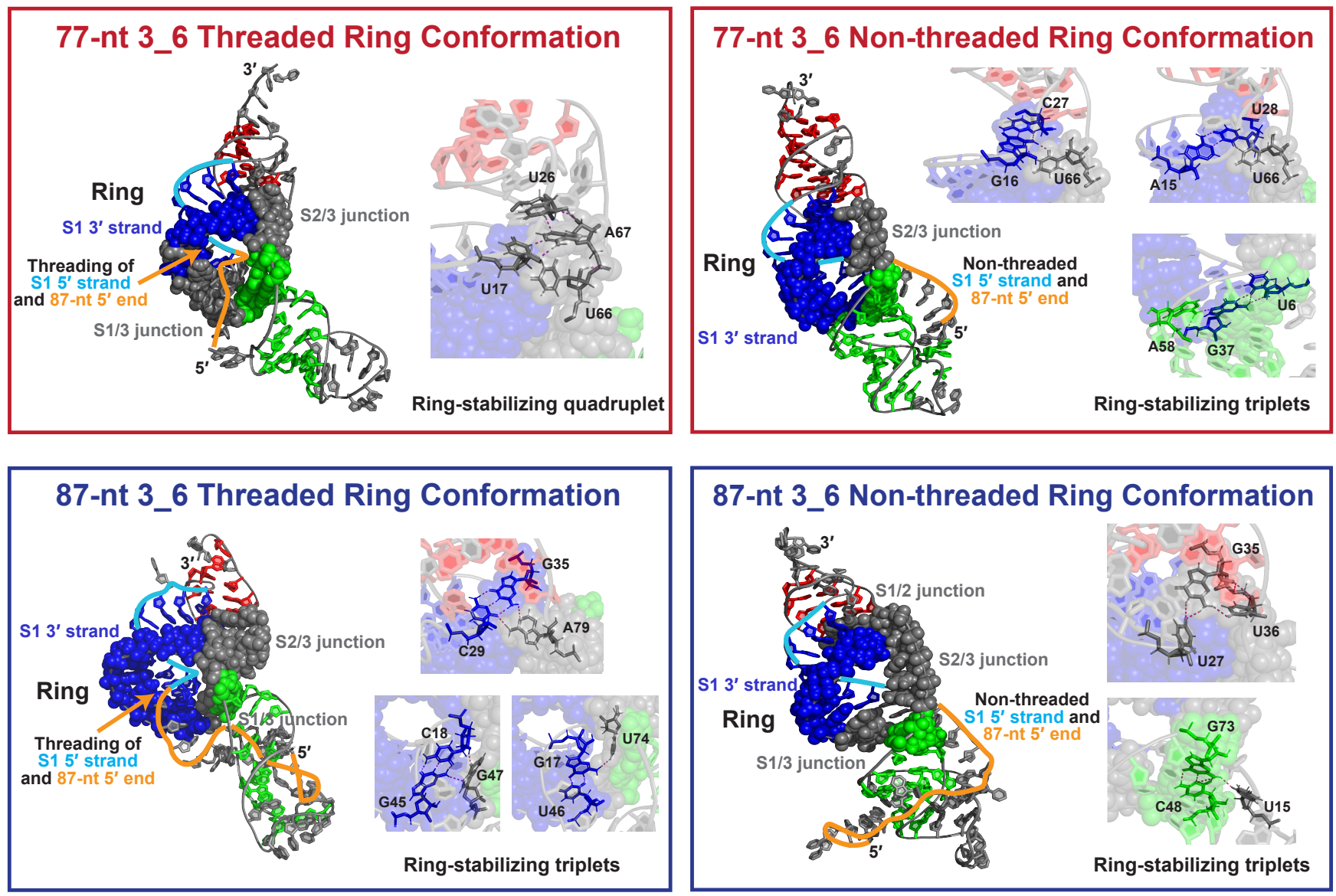

Figure 4: Threaded and non-threaded 3_6 pseudoknot ring conformations. (Top) The threaded 77-nt 3_6 structure (left, RNAComposer), with ring formed by the $3^{\prime}$ strand of Stem 1 , and the Stem 1/3 and 2/3 junctions; the $5^{\prime}$ strand of Stem 1 and the $5^{\prime}$ end thread through the ring. A ring-stabilizing quadruplet formed at the ring top is enlarged. The non-threaded 77-nt 3_6 structure (right, iFoldRNA) has three ring-stabilizing triplets (two at top, one at bottom). (Bottom) Threaded 87-nt (left, RNAComposer) and non-threaded 87-nt (right, iFoldRNA).

$1 / 3$ and $2 / 3$ junctions (G47, U74). In non-threaded systems, 242 triplets form at both the ring top by the $3^{\prime}$ helix end of Stem ${ }_{243}$ 1 and junctions, and the ring bottom by the $5^{\prime}$ helix ends of Stems 1 and 3 .

Comparing our 87-nt threaded 3_6 to the 88-nt Cryo-EM ${ }^{245}$ structure (6.9 $\AA$ resolution), ${ }^{20}$ and our $77-n t$ threaded 3_6 to ${ }^{246}$ the 77-nt Cryo-EM mRNA-ribosome complex (5-7 $\AA),{ }^{21}$ we ${ }^{247}$ find that the experimental "L" shape with coaxially stacked ${ }^{248}$ Stems 1 and 2 are globally similar to our MD structures ${ }^{249}$ (Fig. 5). Our models have narrowed ring holes and shorter 250 Stem 3. In the mRNA-ribosome complex, Stem 3 bends ${ }^{251}$ more towards the S1/S2 coaxial plane. The $5^{\prime}$ end shows ${ }^{252}$ more differences: the $5^{\prime}$ end of the 88-nt Cryo-EM structure forms a small stem-loop, while our $5^{\prime}$ end loosely winds around Stem 3; the $5^{\prime}$ end of the mRNA-ribosome complex ${ }^{254}$ is pulled outward, likely explained by the presence of ribosomal interactions. Overall, our independently developed yet well aligned 3_6 MD structures provide credibility for the following alternative structure modeling.

\section{Alternative 3_3 pseudoknot and 3_5 junction provide insights on structural transitions}

The alternative 3_3 pseudoknot, dominant in our 87 and 144nt FSE constructs, ${ }^{3}$ contains a different Stem 2 formed by the Stem 1 loop and the $5^{\prime}$ end. At 77-nt, the 3_3 pseudoknot has a short Stem 2 (3 base pairs); at 87 and 144-nt, upstream residues form 2 additional base pairs for Stem 2, and also a flanking stem SF with the $3^{\prime}$ end to further seal the conformation (Fig. 6, more details in Fig. S5, S6). Likely due to SF, the 87-nt 3_3 structures have smaller Rg than 77-nt, and they are much more compact than 3_6 (Fig. S3).

The length-dependent interactions in the $3 \_3$ trajectories suggest a potential intermediate mRNA structure that facilitates structural transitions during ribosomal translation and RNA refolding. For 77-nt, the $3^{\prime}$ end residues U74 and U75 form two triplets with two 3_3 Stem 2 base pairs G2-C23 and G3C22 (see Fig. 6). In 3_6, the same end residues U74, U75 base pair with A20 to form Stem 2; in 3_5, they pair with G2 and G1 to form Stem 2. Hence, all three Stem 2 interactions co-exist in this 77-nt 3_3 structure, and this state suggests a starting conformation for a structural transition from $3 \_3$ to 

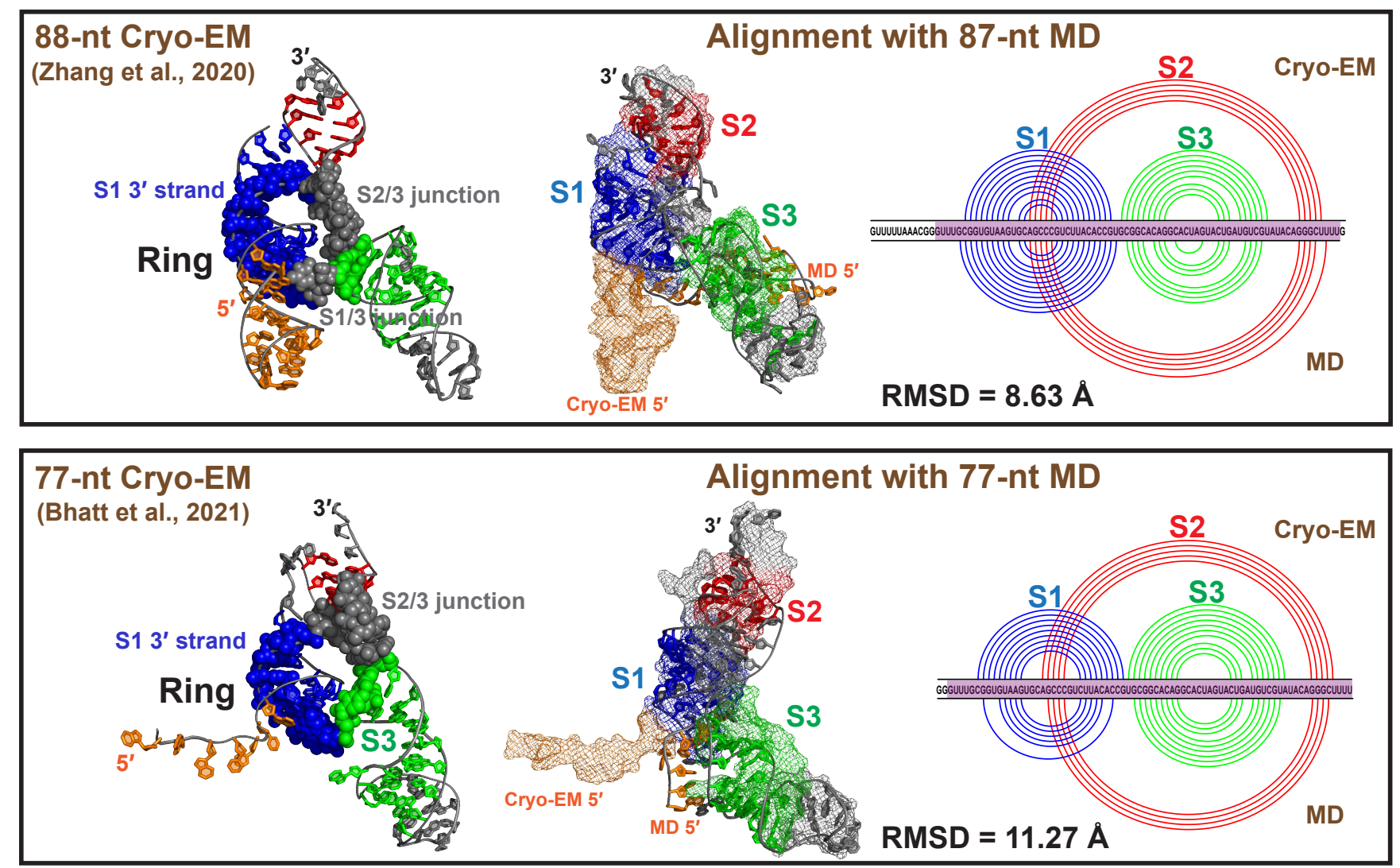

Figure 5: MD 3_6 structures compared to two Cryo-EM structures. (Top, Left) The 88 -nt Cryo-EM structure ${ }^{20}$ in threaded ring conformation is (Middle) aligned with our 87-nt RNAComposer MD structure (Cryo-EM structure in mesh mode, MD in cartoon), with (Right) 2D structural comparisons (Cryo-EM arc plot at top, MD at bottom). The 3D structure alignment is performed by $\mathrm{PyMol}^{48}$ for 75 common residues (highlighted in purple in the 2D plot), and the RMSD is computed. (Bottom) Comparison between the 77-nt Cryo-EM structure ${ }^{21}$ and our 77-nt RNAComposer MD structure.

For the 87 -nt $3 \_3$ systems, the flanking stem SF by the $5^{\prime}$ and $3^{\prime}$ ends blocks alternative Stem 2, and the hydrogen bonding between residue U86 and the Stem 3 base pair C72-G49 maintains the $3^{\prime}$ end away from Stem 2 (Fig. 6). In our 144- ${ }^{28}$ nt models, additional stems form to avoid the mixed Stem 2 triplets (Fig. S5, S6). Hence, all these interactions, espe- 289 cially stem SF must be unwound by the ribosome before the 290 $3^{\prime}$ end is free to form alternative 3_6 Stem 2 (with the Stem 291 1 loop) or 3_5 Stem 2 (with the $5^{\prime}$ end).

Our 3_5 3-way junction RNA at 77-nt is retained in all tra- ${ }^{294}$ jectories (Fig. S7), where the $5^{\prime}$ and $3^{\prime}$ ends base pair to form ${ }_{295}$ Stem 2. This motif has similar Rg and RNA potential energy ${ }_{296}$ to the other two conformations at 77-nt (Fig. S3, S4). A typical 3_5 conformation is elongated in shape as shown in 297 Fig. 6, with Stems 1 and 2 coaxially stacked.

Interactions that impede structural transitions also exist in ${ }_{300}$ the 3.5 structure. The $3^{\prime}$ end residue U77 hydrogen bonds ${ }_{301}$ with Stem 3 base pair A44-U56 to keep Stem 2 near Stem 3302 (Fig. 6). Moreover, the Stem 1/3 junction, the $5^{\prime}$ helix end ${ }_{303}$ of Stem 1, and the Stem $1 / 2$ junction form a triplet and a 304 quadruplet to further lock the Stem 2 orientation and avoid alternative Stem 2. Similar to the 87-nt 3_3, these hydrogen 305 bonds must be broken to allow transition to another motif.

\section{Fluctuations and dominant motions of the three conformations}

Using principal component analysis (PCA), we capture the dominant motion for 3_6 to be a structural switch between the "L" and the linear shape, via bending of Stem 3 (Fig. 7). The pseudoknot complex (Stems 1 and 2) and the ring conformation are maintained throughout this motion, as does the ring-holding triplet at the bottom. Longer 3_6 structures tend to remain linear, with upstream and downstream stems moving more substantially (Fig. S8).

Consistent with the above motions, we see a peak in the 3_6 root mean square fluctuations (RMSF) in the Stem 3 loop region for all lengths (Fig. 7). The unpaired $3^{\prime}$ end also exhibits high RMSF, especially for 77-nt, as no downstream pairs restrict its movement. The RMSF, average number of hydrogen bonds (H-bond), and the interaction energies all indicate that Stem 1 is the strongest, followed by Stem 3, and lastly by Stem 2 (Fig. S9, S10).

The 3_3 pseudoknot's dominant motion is contraction and 

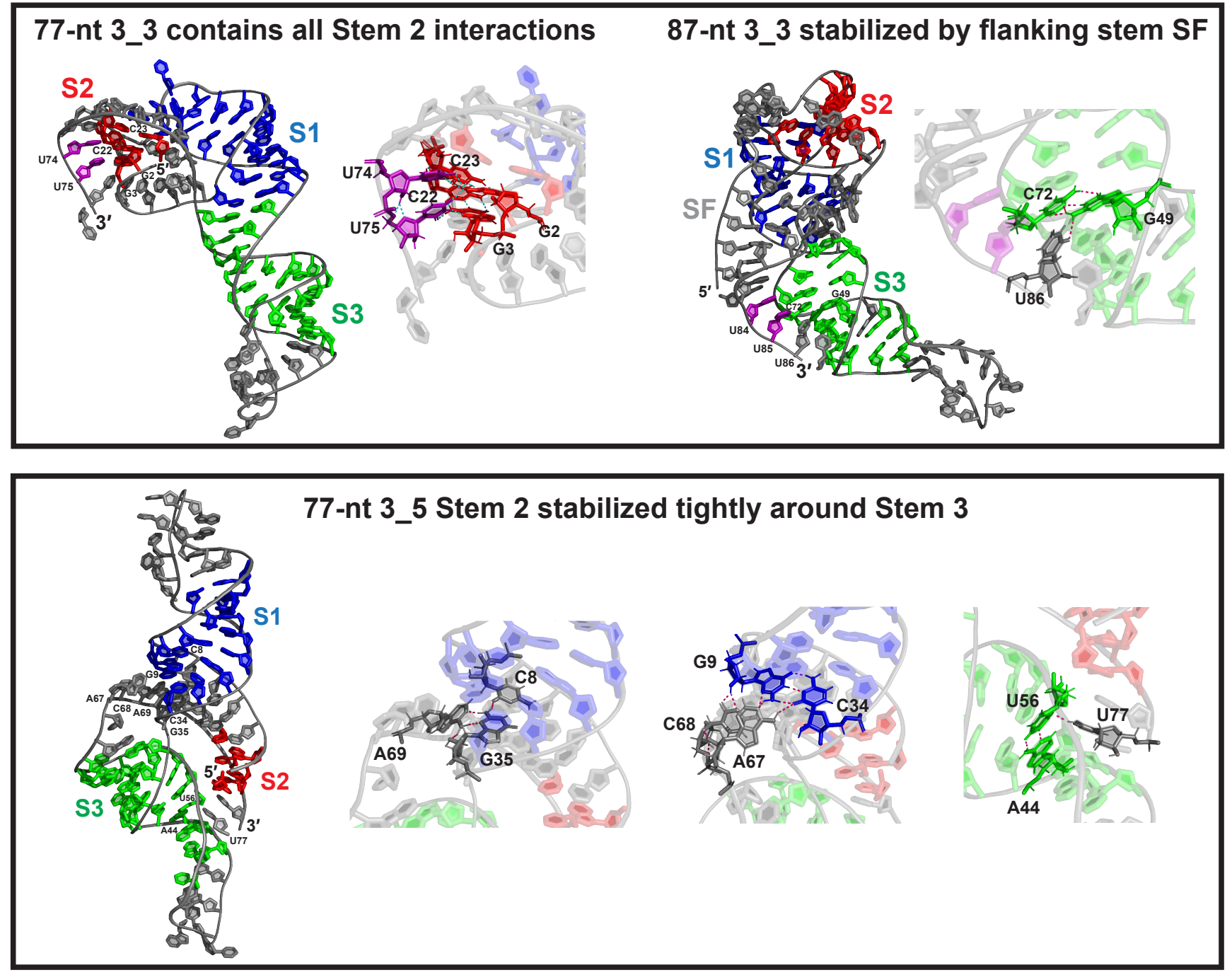

Figure 6: Alternative 3_3 and 3_5 conformations. (Top) The 77 and 87-nt 3_3 pseudoknot structures (iFoldRNA). For 77-nt, two residues in the $3^{\prime}$ end (purple), which are involved in the 3_6 and 3_5 Stem 2, form triplets with the 3_3 Stem 2. For 87-nt, the $5^{\prime}$ and $3^{\prime}$ end bind to form flanking stem SF, and the same two $3^{\prime}$ end residues (purple) are locked around Stem 3 by a downstream triplet. (Bottom) The 3_5 junction 77-nt MD model (SimRNA). The Stem 2 helix formed by the $5^{\prime}$ and $3^{\prime}$ ends is stabilized around Stem 3 by multiple hydrogen bonds.

stretching caused by the bending of $3^{\prime}$ end and Stem 3 loop 322 (Fig. 7, Fig. S11). In this motion, Stems 1 and 2, especially ${ }_{32}$ triplets that contain interactions from all three Stem 2 (pur- ${ }_{324}$ ple and red residues in Fig. 7), are stable and move in unison. ${ }_{325}$ That these triplets are not transient suggests that they may be 326 part of the structural transition among alternative conforma- ${ }_{327}$ tions, as discussed above.

Comparing to 3_6, we see a higher RMSF peak value in the 3_3 Stem 3 loop region, and more fluctuations in 3_3 Stem $3 з 0$ 1 region due to the pseudoknot bending, with a consistent ${ }_{331}$ lower Stem 1 H-bond number (Fig. S9). A clear jump occurs 332 for 3_3 Stem $2 \mathrm{H}$-bond number, when the length increases from 77 to 87 -nt, resulting in a stronger Stem 2 of 3_3 than 334 3_6 (Fig. S9), following our finding of dominant 3_6 at 77-nt 335 while dominant 3_3 at 87-nt. ${ }^{3}$ A similar trend is observed for the stem interaction energy (Fig. S10).
For the 3_5 junction, Stem 1 twisting is dominant (Fig. 7, Fig. S12): as Stem 1 twists backwards, it pulls the downstream backbone and hence Stem 3 moves up towards Stem 1. All the triplets and hydrogen bonds that lock the Stem 2 orientation (Fig. 6) are maintained, and Stem 2 is kept near Stem 3 while they move together. Peak RMSF in the loop regions of Stems 1 and 3, and low values in the $5^{\prime}$ and $3^{\prime}$ ends are notable.

Overall, all three conformations have stable Stem 1, flexible Stem 3 loop, and relatively stable Stem 2 regions. The triplets and hydrogen bonds are mostly maintained throughout the simulations, and this helps stabilize key features such as the ring of 3_6 and the combined Stem 2 interactions in 3_3. 

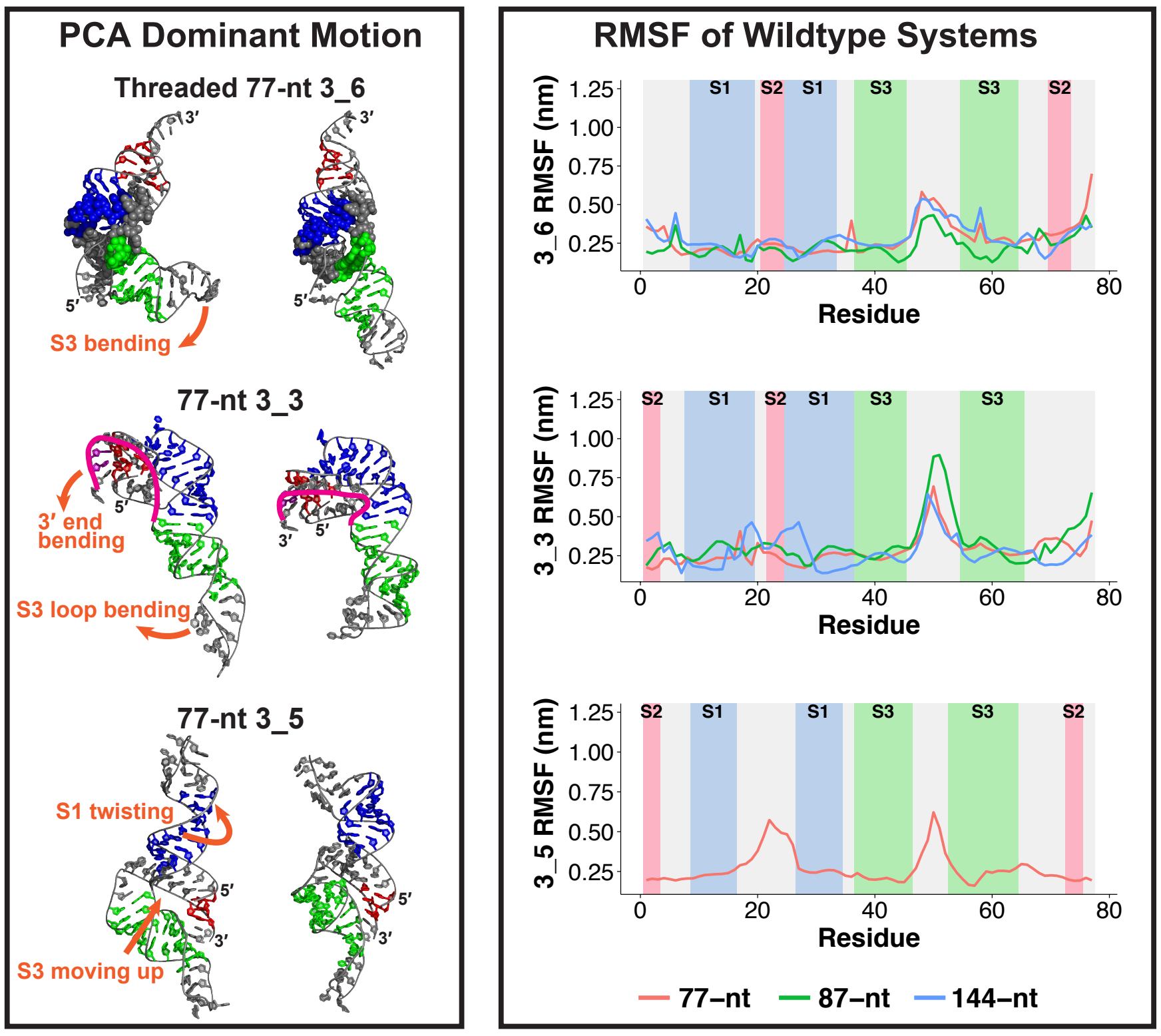

Figure 7: Dynamic analysis of the wildtype 3_6, 3_3, and 3_5 systems. (Left) Dominant motions of the threaded 77-nt 3_6 pseudoknot (RNAComposer), 77-nt 3_3 pseudoknot (iFoldRNA), and 77-nt 3_5 junction (SimRNA) extracted by principal component analysis (PCA). (Right) Flexibility of the three conformations as reflected by root mean square fluctuations (RMSF). For the 3_6 and 3_3 pseudoknots, the RMSF is shown for the common 77-nt region at various lengths; for 3_5 junction, RMSF at 77-nt. The different stem regions are colored and labeled.

\section{Minimal mutations stabilize the three confor- ${ }_{347}$ mations}

Our predicted mutations confirmed by SHAPE probing were designed to suppress conformational transitions and stabilize specific conformations over all alternatives, for the 77 and 144-nt 3_6 pseudoknot, 77-nt 3_3 pseudoknot, and 77-nt 3 5 junction. ${ }^{3,25}$ Our dynamics analyses below of these mutants compared to the wildtype trajectories help interrogate the mechanisms and consequences of structural stability; we use the same representative mutant systems in Fig. 8 as for the wildtype, except for 77-nt 3_6.
The 6 mutations in the 77-nt 3_6 pseudoknot-strengthening mutant (PSM) include 4 mutations ([G18A, C19A, C68A, A69C]) that lengthen Stem 2 by up to 4 base pairs (Table 1) and 2 mutations at the $5^{\prime}$ end to exclude alternative 3_3 and 3_5 Stem 2. Because the SimRNA mutant has the longest Stem 2 ( 9 base pairs), we compare it to the corresponding wildtype. We observe a dramatic transformation from " $\mathrm{L}$ " shape (wildtype) to a linear shape (Fig. 8). Indeed, all 3_6 mutant systems adopt this linear shape, and the structural switch between the two shapes has been suppressed (Fig. S13, S14).

For the 144-nt 3_6 PSM, one additional mutation in the downstream region suppresses formation of competing 
Table 1: Comparison of the motif-strengthening mutants and the wildtype systems. For each mutant, the mutations, the 3D prediction programs ( $R$ for RNAComposer, $S$ for SimRNA, $I$ for iFoldRNA, $V$ for Vfold3D), the wildtype and mutant Stem 2 lengths, and the newly formed Stem 2 base pairs involving the mutated residues are listed.

\begin{tabular}{|c|c|c|c|}
\hline Program & WT S2 & Mutant S2 & Base pairs involving mutations \\
\hline \multicolumn{4}{|c|}{ 77-nt 3_6 PSM [G3U, U4A, G18A, C19A, C68A, A69C] } \\
\hline $\mathrm{R}$ & 4 & 4 & G25-C69 \\
\hline $\mathrm{S}$ & 4 & 9 & A18-U76, A19-U75, G25-C69, U26-A68 \\
\hline I & 7 & 7 & A19-U75, G25-C69 \\
\hline $\mathrm{V}$ & 4 & 8 & A18-U76, A19-U75, G25-C69, U26-A68 \\
\hline \multicolumn{4}{|c|}{ 144-nt 3_6 PSM [G40U, U41A, G55A, C56A, C105A, A106C, C137A] } \\
\hline $\mathrm{R}$ & 4 & 5 & G62-C106 \\
\hline $\mathrm{I}$ & 5 & 4 & A56-U112 \\
\hline \multicolumn{4}{|c|}{ 77-nt 3_3 PSM [U4C, G71A, G72U] } \\
\hline I & 3 & 7 & C4-G21 \\
\hline \multicolumn{4}{|c|}{ 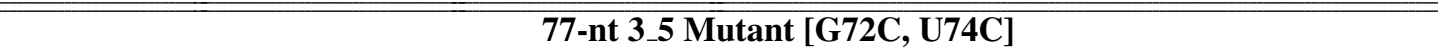 } \\
\hline $\mathrm{R}$ & 3 & 7 & G1-C74, G3-C72 \\
\hline $\mathrm{S}$ & 3 & 7 & G1-C74, G3-C72 \\
\hline I & 4 & 7 & G1-C74, G3-C72 \\
\hline $\mathrm{V}$ & 3 & 6 & G3-C72 \\
\hline
\end{tabular}

stems. ${ }^{3}$ The central 3_6 pseudoknot region aligns well be- ${ }^{394}$ tween the wildtype and mutant systems, both adopting the 395 linear shape (Fig. 8, Fig. S13). The major difference oc- 396 curs in the upstream region: in the wildtype, upstream and downstream stems form on the same side of the central 3_6 pseudoknot; in the mutant, they are on different sides, due to our [G40U, U41A] mutations. From PCA, we see a relatively stable central 3_6 pseudoknot, while quite flexible upstream and downstream stems in the mutant (Fig. S14). As both our 77 and 144-nt 3_6 mutants adopt linear con- ${ }_{399}$ formations, we hypothesize that this may be a more stable ${ }_{400}$ conformation, by separating the $5^{\prime}$ and $3^{\prime}$ ends further away ${ }_{401}$ from each other to avoid alternative 3_3 and 3_5 Stem 2.

In our 77-nt 3_3 PSM, a large increase of Stem 2 length from 3 to 7 base pairs is induced by mere three mutations [U4C, G71A, G72U] (Table 1, Fig. S15). The first mutation enhances the 3_3 Stem 2 and the others avoid alternative 3_6 and 3.5 motifs. The main structural changes are a vertical $5^{\prime}$ end between the Stem 1 loop and helix instead of staying 408 horizontal below, compact Stems 1 and 2, and elimination of ${ }_{409}$ triplets formed by the $3^{\prime}$ end with Stem 2 (Fig. 8). Hence, ${ }_{410}$ our mutations stabilize the 3_3 conformation without alter- ${ }_{41}$ native Stem 2 interactions. The dominant motion occurs in ${ }_{412}$ the Stem 3 region (Fig. S16).

Our 77-nt 3_5 mutant with only 2 mutations [G72C, U74C] also enjoys a considerable enhancement of Stem 2 from 3-4 base pairs to 6-7 (Table 1, Fig. S17). The three stems then have similar sizes (Fig. 8). Stem 2 is no longer held around Stem 3, but instead extends as a third helical arm. Coaxial stacking of Stems 1 and 2, as well as a tilting motion of these two stacked stems, are observed (Fig. S18).

Overall, our enhanced Stem 2 in the three mutants leads to dramatic structural changes, especially for the 77-nt 3_6 and $3 \_5$ systems. PCA analysis reveals stabilization of the lin- ear shape in 3_6 PSM, thereby eliminating the "L" to linear shape switch. For the 77-nt 3_3 mutant, triplets associated with possible structural transitions are also eliminated.

\section{Discussion: Implications to frameshifting and anti-viral strategies}

Our microsecond MD simulations of three possible conformations of the SARS-CoV-2 FSE, namely 3_6 pseudoknot, 3_3 pseudoknot, and 3.5 junction for different lengths (Fig. 1, 2), highlight different structural features and motions. Our motif-strengthening mutant trajectories clarify how these mutations alter the RNA conformations and motions (Fig. 8). The combined insights suggest three anti-viral intervention avenues and a mechanism for frameshifting that links our three alternative conformations (Fig. 9).

The first anti-viral approach is to alter the 3_6 pseudoknot plasticity. Pseudoknot stabilizing hydrogen bonds are identified at Stem 1/2 and 2/3 junctions of 3_6 (Fig. 3). Since conformational plasticity has a large impact on frameshifting efficiency, ${ }^{9}$ mutating these residues to further strengthen or destroy the pseudoknot should interrupt the frameshifting process. Indeed, Bhatt et al. achieve a significant reduction in frameshifting efficiency by mutating these junctions. ${ }^{21}$ In our prior SHAPE probing, 3_6 Stem 2 enhancing mutations in this region modify the conformational landscape to $100 \% 3 \_6 .{ }^{3}$ Both studies underscore the sensitivity of the 3_6 pseudoknot and its associated frameshifting to these junction residues, which define good targets for CRISPR gene-editing (Fig. 9, left).

The second approach is to strengthen the $5^{\prime}$ end threading in the 3_6 ring conformation. The ring is formed by the $3^{\prime}$ strand of Stem 1, the Stem $1 / 3$ and $2 / 3$ junctions, and are 

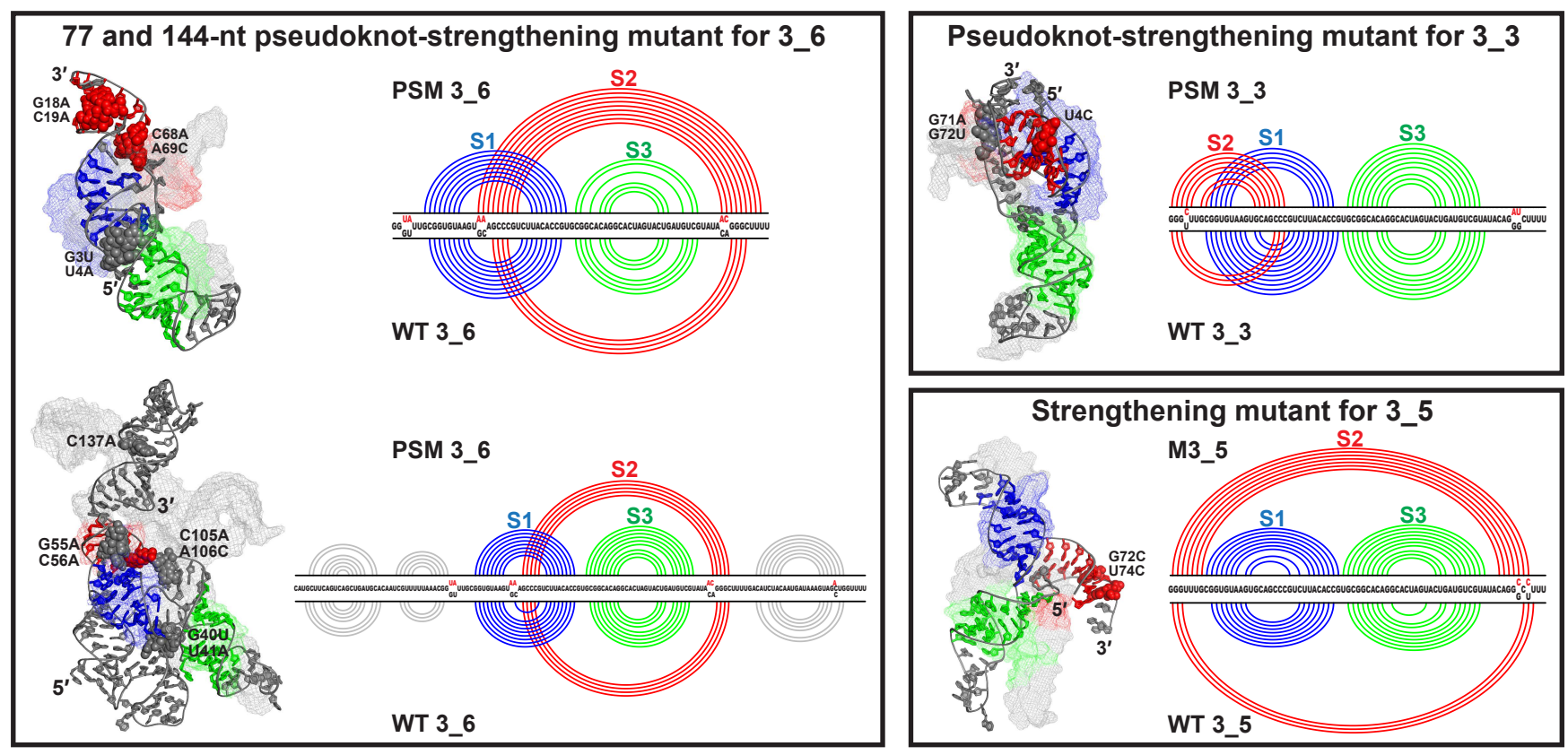

Figure 8: Comparison of the motif-strengthening mutants with the wildtype systems. For 3_6 pseudoknot, both the 77-nt (SimRNA) and 144-nt (RNAComposer) PSM are shown in cartoon mode with their wildtype systems aligned in mesh (by $\mathrm{PyMol}^{48}$ for the 77-nt region). The mutations are highlighted as spheres in PSM structure and labeled. The 2D structure comparison is also provided with PSM at top and wildtype at bottom. Comparisons for the 77-nt 3_3 PSM (iFoldRNA) and 77-nt 3_5 mutant (SimRNA) are shown in similar manner.

stabilized by hydrogen bonding and base triplet interactions 455 (Fig. 4). In some systems, the $5^{\prime}$ strand of Stem 1 and the 456 $5^{\prime}$ end thread through the ring, which probably resists ribo- ${ }^{457}$ somal unwinding ${ }^{20}$ by requiring a higher unfolding force; ${ }^{49}{ }_{458}$ thus, strengthening the threading may increase the mechan- 459 ical barrier for translation. Recently, two alkaloids (eme- 460 tine and cephaeline) predicted to bind the threading initiation ${ }_{461}$ site were found to inhibit SARS-CoV-2 viral replication. ${ }^{50}$

Hence, the $3^{\prime}$ helix end of Stem 1, which we find to close the ${ }^{462}$ ring and initiate threading, defines a target binding region to ${ }^{463}$ impede ribosomal translation (Fig. 9).

The third approach is to target the 3_6 pseudoknot structural 466 switch between an "L" shape (coaxially stacked Stems 1 and ${ }_{467}$ 2 and an extruding Stem 3) and a linear shape (vertical stack- ${ }_{468}$ ing of the 3 stems), revealed by our PCA analysis (Fig. 7). 469 In the mRNA-ribosome Cryo-EM structure captured during 470 translation, ${ }^{21}$ the "L" shaped 3_6 wedges at the mRNA en- 471 try channel and resists unwinding by the helicase, which 472 generates tension on the upstream mRNA. ${ }^{21}$ This structural switch might then enhance fluctuations of this tension and 473 send frameshifting signals to the ribosome. When switching 474 from the "L" to linear shape, residues in the Stem $2 / 3$ junc- 475 tion are exposed (Fig. 7); small molecules like MTDB ${ }^{10,51} 476$ can thus block the switch and hamper frameshifting (Fig. 9). ${ }^{477}$ Another option is to deploy our 3_6 mutant, which assures a 478 stabilized linear shape (Fig. 8).

Overall, by analyzing the hydrogen bonding interactions and motions of different 3_6 systems, we offer three strate- ${ }_{482}$ gic anti-viral targeting regions: the $3^{\prime}$ helix end of Stem ${ }^{483}$ 1 and Stem $1 / 2$ and 2/3 junction residues (Fig. 9). Al- 484 though several drugs/small molecules have been shown to inhibit SARS-CoV-2 frameshifting, including $M T D B,{ }^{18,51,52}$ alkaloids, ${ }^{50}$ Merafloxacin, ${ }^{53}$ Ivacaftor, and Huperzine A, ${ }^{54}$ they are mainly found by high-throughput drug screening, so the underlying inhibition mechanism is unexplained and, in some cases, the binding regions are unknown. Our targeting regions above emerged from mechanistic considerations.

Furthermore, based on interactions analyzed in our trajectories of different lengths (Fig. 6), we propose a possible FSE structural transition pathway (Fig. 9, right): during translation, when the ribosome is far away from the FSE region, the dominant conformation is a 3_3 with stem SF; as the ribosome approaches and occludes the slippery site, stem SF is unwound, and the $3^{\prime}$ end moves to the 3_3 Stem 2 region to form the triplets and structural transition to 3_6 or 3_5 begins; when the ribosome further elongates, the $5^{\prime}$ end (including the slippery site) becomes completely occluded, and only $3 \_6$ remains viable.

This structural transition pathway may be associated with SARS-CoV-2 regulatory functions, as RNA structural alterations can lead to different biological outcomes. ${ }^{55}$ For example, ribosomal RNA (rRNA) samples alternate structures to control translation. ${ }^{56}$ The timescale at which the transitions occur depends on the scale of conformational rearrangements. Interhelical or loop dynamics occur on picosecond to microsecond timescale. Base pairing or tertiary structure changes occur on microsecond to second range. Major interconversions between secondary structures occur on millisecond and longer. ${ }^{57}$ Given that the ribosome pauses $\sim 2.8 \mathrm{~s}$ between translocations, ${ }^{58}$ this time allows for the structural 

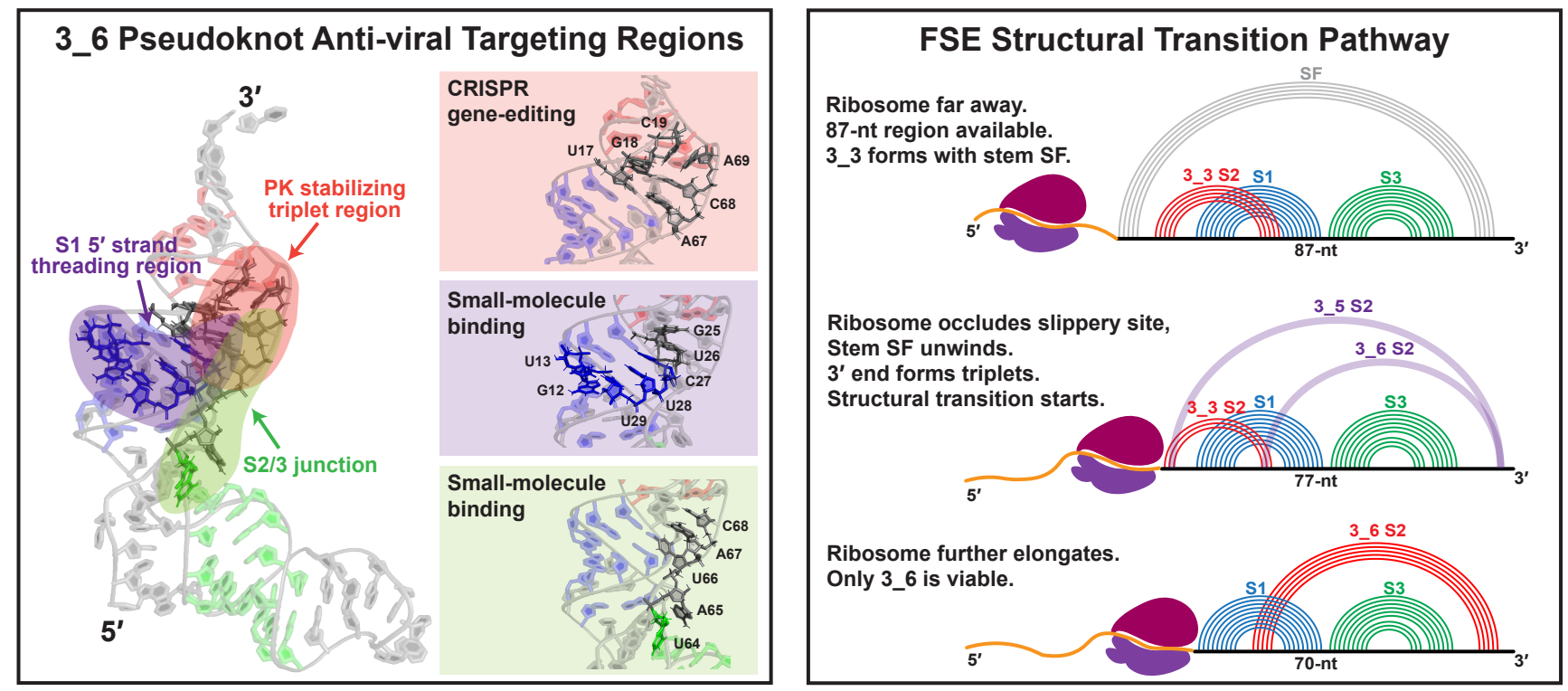

Figure 9: Implications of the unraveled structures and motions to anti-viral therapeutics and frameshifting mechanisms. (Left) Anti-viral target regions in the 3_6 pseudoknot. (Right) Proposed structural transition pathway for the SARS-CoV-2 frameshifting element.

switches and transitions discussed here to occur.

In sum, our microsecond MD simulations extend beyond ${ }_{514}$ consistent 3D structure models for the prevalent 3_6 pseu- ${ }_{515}$ doknot in literature, ${ }^{3,17-22,24,25,38,59}$ by providing the first ${ }_{516}$ 3D models for the alternative FSE structures and the motif- ${ }_{517}$ strengthening mutants, which were verified by SHAPE ex- ${ }_{518}$ periments. ${ }^{3}$ We suggest several potential interventions to ${ }_{519}$ interfere with SARS-CoV-2 frameshifting and ribosomal ${ }_{520}$ translation, and provide insights into frameshifting mecha- ${ }_{52}$ nism (Fig. 9). These ideas offer anti-viral strategies against Covid-19 by small-molecule binding and CRISPR geneediting. More broadly, our methods and analyses extend to other viral systems. Together with other computational and experimental studies, we hope to advance our understanding of the basic science associated with complex frameshifting ${ }^{523}$ mechanisms and therapeutic applications.

\section{Materials and Methods}

\section{RAG Notation and Mutations}

In our RNA-As-Graphs (RAG) framework, RNA secondary structures containing pseudoknots are represented as dual ${ }^{533}$ graphs. ${ }^{27}$ Each stem ( $\geq 2$ base pairs) denotes a vertex, and ${ }^{534}$ every single strand or loop is an edge (hairpins are self-loops; ${ }^{53}$ 1-nt bulges, internal loops with two 1-nt strands, and dan- ${ }^{536}$ gling ends are ignored). Every non-isomorphic dual graph is ${ }^{537}$ assigned an identifier $V \_n$, where $V$ is the vertex number and ${ }^{538}$ $n$ is a unique motif identifier. Our dual graph library consists of over 100,000 unique dual graphs for 2-9 vertices. ${ }^{30}$ oped our inverse folding program RAG-IF modified for dual graphs. ${ }^{25,36}$ For manually selected mutation regions and a target 2D structure, RAG-IF uses a genetic algorithm to generate a pool of candidate RNA sequences with mutations. These candidates are screened by 2D prediction programs to ensure the correct graph folding, and are optimized for minimal mutations. Detailed design of the mutants is described in. 3,25

\section{FSE Lengths and Conformations}

We model the FSE structure at three sequence lengths: 77nt without the 7-nt slippery site, 87-nt with the slippery site plus 3 additional residues at the $5^{\prime}$ end, and 144-nt with the slippery site plus 30 additional residues at each end. We perform MD simulations for all three conformations for the 77nt FSE. (Even though the 3_3 pseudoknot was not observed at this length, we study it for comparison with other lengths.) For 87 and 144-nt, we model the 3_6 and the 3_3 conformations, with additional stems formed by the upstream and downstream nucleotides (Fig. 1).

Besides wildtype FSEs, we also model four motifstrengthening mutants predicted previously: ${ }^{3,25}$ 77-nt 3_6 PSM with 6 mutations [G3U, U4A, G18A, C19A, C68A, A69C], 144-nt 3_6 PSM with an additional mutation C137A, 77-nt 3_3 PSM with 3 mutations [U4C, G71A, G72U], and 77-nt 3_5 mutant with 2 mutations [G72C, U74C]. 


\section{D and 3D FSE Structures}

The 2D structure of the wildtype 77-nt 3_6 pseudoknot was predicted by PKNOTS, ${ }^{60}$ and all other $2 \mathrm{D}$ conformations were modeled by ShapeKnots with SHAPE reactivities incorporated. ${ }^{3,61}$

Corresponding 3D structures were predicted, with the se- 597 quences and the 2D structures as input using RNACom- 598 poser, ${ }^{62}$ Vfold3D, ${ }^{63}$ SimRNA, $^{64}$ and iFoldRNA ${ }^{65}$ for $77{ }^{599}$ and 87-nt, and RNAComposer, iFoldRNA, and Farfar ${ }^{66}$ for ${ }^{600}$ 144-nt, as SimRNA and Vfold3D failed to produce models for this length (see Table S1). For 3D structure prediction programs that gave multiple structures as output, the first structure that retained the correct motif was selected for MD simulations.

\section{Molecular Dynamics Details}

The MD simulation protocol follows our prior work. ${ }^{25} \mathrm{We}$ use Gromacs 2020.3 and $2020.4,{ }^{47}$ with the Amber OL3 forcefield. ${ }^{67}$ The systems are solvated in the cubic box with TIP3P water model, with a buffer of $10 \AA$ from the RNA molecule. ${ }^{68}$ The systems are first neutralized with sodium ions and set to a $0.1 \mathrm{M} \mathrm{NaCl}$ bulk concentration with additional $\mathrm{Na}^{+}$and $\mathrm{Cl}^{-}$ions. The systems are energy minimized via steepest descent and equilibrated under NVT (300 K) and NPT (1 bar and $300 \mathrm{~K}$ ) ensembles for 100 ps each. Sim- ${ }_{613}$ ulations are run with a timestep of 2 fs and a SHAKE-like ${ }_{614}$ LINCS algorithm ${ }^{69}$ with constraints on all atom bonds. The Particle Mesh Ewald method ${ }^{70}$ is used to treat long-range 615 electrostatics. Production runs are performed for $1 \sim 1.5 \mu \mathrm{s}{ }_{616}$ under NPT to ensure stable RMSD. Structures from the last $500 \mathrm{~ns}$ of each simulation are used for analysis.

Clustering is performed on frames every 200 ps for RNA ${ }_{619}$ non-H backbone atoms, using the Gromos clustering method 620 with $2,2.5,3$, and $3.5 \AA$ cutoffs. The largest cluster center structures (cutoff of $2.5 \AA$ for 77 -nt and 87 -nt systems or $3.5 \AA$ for $144-n t$ systems) are extracted from MD simulations to show and analyze in Results and Supplementary Information. The cutoffs are chosen to ensure that all simulations for 622 the same dual graph topology produce a feasible number of ${ }^{623}$ clusters with outlier structures excluded. See Fig. S19 for ${ }_{624}$ more details. PCA is performed on structures every 250 ps. Cluster ${ }^{627}$ analysis, PCA motion analysis, calculations of Rg, RMSF, 628 RNA potential energy, interaction energy (sum of short-term ${ }_{629}$ Lennard-Jones and Coulomb interactions) between the two ${ }^{630}$ strands within each stem, and the number of hydrogen bonds ${ }^{631}$ in each stem are performed via Gromacs 2020.3. ${ }^{47}$ The 2D ${ }_{632}$ structures, base pairing, and stacking information are ana- 633 lyzed using 3DNA-DSSR. ${ }^{46}$ The structure alignment is per- ${ }^{634}$ formed using $\mathrm{PyMol}^{48}$ align with RMSD computed. Prince or Greene supercomputer clusters at the New York ${ }^{638}$
University High Performance Computing facilities. Each compute node in the Prince cluster is equipped with two Intel Xeon E5-2690v4 2.6 GHz CPUs ("Broadwell," 14 cores/socket, 28 cores/node) and 125 GB memory. Each simulation is performed with seven to eight dedicated nodes (i.e., 196-224 cores), so the simulations complete in 7-10 days. Each compute node in the Greene clusters is equipped with two Intel Xeon Platinum 8268 24C 205W $2.9 \mathrm{GHz}$ CPUs with 48 cores/node and 192 GB memory. Each simulation is performed with 30 nodes using 32 cores each, so that the simulations complete in 2-4 days.

\section{Acknowledgments}

General: We thank Shereef Elmetwaly for technical assistance and David Ackerman, Stratos Efstathiadis, and Shenglong Wang from the NYU High-Performance Computing facilities for providing our group dedicated resources to perform this work.

Funding: We gratefully acknowledge funding from the National Science Foundation RAPID Award 2030377 from the Division of Mathematical Science and the Division of Chemistry, National Institutes of Health R35GM122562 Award from the National Institute of General Medical Sciences, and Philip Morris International to T.S.

Author contributions: All authors contributed to data analysis, project design, and manuscript preparation.

Competing interests: The authors declare that they have no competing interests.

Data and materials availability: All data needed to evaluate the conclusions in the paper are present in the paper and/or the Supplementary Materials. Additional data related to this paper may be requested from the authors.

\section{References}

(1) Jacks, T.; Varmus, H. Expression of the Rous sarcoma virus pol gene by ribosomal frameshifting. Science 1985, 230, 1237-1242.

(2) Brierley, I.; Boursnell, M.; Binns, M.; Bilimoria, B.; Blok, V.; Brown, T.; Inglis, S. An efficient ribosomal frame-shifting signal in the polymerase-encoding region of the coronavirus IBV. EMBO J. 1987, 6, 3779-3785.

(3) Schlick, T.; Zhu, Q.; Dey, A.; Jain, S.; Yan, S.; Laederach, A. To Knot or Not to Knot: Multiple Conformations of the SARS-CoV-2 Frameshifting RNA Element. J. Amer. Chem. Soc. 2021, 143, 1140411422 .

(4) Kelly, J.; Woodside, M.; Dinman, J. Programmed 1 Ribosomal Frameshifting in coronaviruses: A therapeutic target. Virology 2021 $554,75-82$.

(5) Dinman, J.; Ruiz-Echevarria, M.; Czaplinski, K.; Peltz, S. Peptidyltransferase inhibitors have antiviral properties by altering programmed -1 ribosomal frameshifting efficiencies: Development of model systems. Proc. Nat. Acad. Sci., USA 1997, 94, 6606-6611. 
(6) Kinzy, T.; Harger, J.; Carr-Schmid, A.; Kwon, J.; Shastry, M.; Jus- 701 tice, M.; Dinman, J. New Targets for Antivirals: The Ribosomal A- 702 Site and the Factors That Interact with It. Virology 2002, 300, 60-70. 703

(7) Lopinski, J.; Dinman, J.; Bruenn, J. Kinetics of ribosomal pausing during programmed -1 translational frameshifting. Mol. Cell. Biol. 705 2000, 20, 1095-1103.

(8) Namy, O.; Moran, S.; Stuart, D.; Gilbert, R.; Brierley, I. A mechanical explanation of RNA pseudoknot function in programmed ribosomal 708 frameshifting. Nature 2006, 441, 244-247.

(9) Ritchie, D.; Foster, D.; Woodside, M. Programmed -1 frameshifting efficiency correlates with RNA pseudoknot conformational plasticity, not resistance to mechanical unfolding. Proc. Nat. Acad. Sci., USA 712 2012, 109, 16167-16172.

(10) Ritchie, D.; Soong, J.; Sikkema, W.; Woodside, M. Anti-frameshifting Ligand Reduces the Conformational Plasticity of the SARS Virus 7 Pseudoknot. J. Amer. Chem. Soc. 2014, 136, 2196-2199.

(11) Kim, H.; Liu, F.; Fei, J.; Bustamante, C.; Gonzalez, R.; Tinoco, I. A frameshifting stimulatory stem loop destabilizes the hybrid state and impedes ribosomal translocation. Proc. Nat. Acad. Sci., USA 2014, $111,5538-5543$.

(12) Chen, J.; Petrov, A.; Johansson, M.; Tsai, A.; O’Leary, S.; Puglisi, J. Dynamic pathways of -1 translational frameshifting. Nature 2014, $512,328-332$.

(13) Caliskan, N.; Katunin, V.; Belardinelli, R.; Peske, F.; Rodnina, M Programmed -1 Frameshifting by Kinetic Partitioning during Impeded Translocation. Cell 2014, 157, 1619-1631.

(14) Brierley, I.; Pennell, S.; Gilbert, R. Viral RNA pseudoknots: versatile motifs in gene expression and replication. Nat. Rev. Microbiol. 2007, $5,598-610$.

(15) Parkin, N.; Chamorro, M.; Varmus, H. Human immunodeficiency virus type 1 gag-pol frameshifting is dependent on downstream mRNA secondary structure: demonstration by expression in vivo. $\mathrm{Vi}$ rol. J. 1992, 66, 5147-5151.

(16) Brierley, I; Digard, P.; Inglis, S. Characterization of an efficient coro- 733 navirus ribosomal frameshifting signal: Requirement for an RNA pseudoknot. Cell 1989, 57, 537-547.

(17) Wacker, A.; Weigand, J.; Akabayov, S.; Altincekic, N.; Bains, J., et al. Secondary structure determination of conserved SARS-CoV-2 RNA elements by NMR spectroscopy. Nucleic Acids Res. 2020, 48, 12415- ${ }^{737}$ 12435 .

(18) Kelly, J.; Olson, A.; Neupane, K.; Munshi, S.; Emeterio, J.; Pol- 739 lack, L.; Woodside, M.; Dinman, J. Structural and functional conser- 740 vation of the programmed -1 ribosomal frameshift signal of SARS 741 coronavirus 2 (SARS-CoV-2). J. Biol. Chem. 2020, 295, 10741- ${ }^{742}$ 10748 .

(19) Lan, T; Allan, M; Malsick, L; Khandwala, S ; Nyeo, S. 744 et al. Insights into the secondary structural ensembles of the 745 full SARS-CoV-2 RNA genome in infected cells. bioRxiv, doi: ${ }^{746}$ 10.1101/2020.06.29.178343, preprint posted February 2021.

20) Zhang, K.; Zheludev, I.; Hagey, R.; Haslecker, R., et al. Cryo-EM and 748 antisense targeting of the 28-kDa frameshift stimulation element from 749 the SARS-CoV-2 RNA genome. Nat. Struct. Mol. Biol. 2021 ,

(21) Bhatt, P.; Scaiola, A.; Loughran, G.; Leibundgut, M.; Kratzel, A., et al. 751 Structural basis of ribosomal frameshifting during translation of the 752 SARS-CoV-2 RNA genome. Science 2021, 372, 1306-1313.

(22) Ziv, O.; Price, J.; Shalamova, L.; Kamenova, T.; Goodfellow, I.; Weber, F.; Miska, E. The Short- and Long-Range RNA-RNA Interactome 755 of SARS-CoV-2. Mol. Cell 2020, 80, 1067-1077.e5.

23) Roman, C.; Lewicka, A.; Koirala, D.; Li, N.; Piccirilli, J. The SARSCoV-2 Programmed -1 Ribosomal Frameshifting Element Crystal 758 Structure Solved to $2.09 \AA$ Using Chaperone-Assisted RNA Crystal- 759 lography. ACS Chem. Biol. 2021, 16, 1469-1481.
(24) Omar, S.; Zhao, M.; Sekar, R.; Moghadam, S.; Tuszynski, J.; Woodside, M. Modeling the structure of the frameshift-stimulatory pseudoknot in SARS-CoV-2 reveals multiple possible conformers. PLOS Comput. Biol. 2021, 17, e1008603.

(25) Schlick, T.; Zhu, Q.; Jain, S.; Yan, S. Structure-Altering Mutations of the SARS-CoV-2 Frameshifting RNA Element. Biophys. J. 2021, 120, 1040-1053.

(26) Rangan, R.; Watkins, A.; Chacon, J.; Kladwang, W.; Zheludev, I., et al. De novo 3D models of SARS-CoV-2 RNA elements from consensus experimental secondary structures. Nucleic Acids Res. 2021, 49, 3092-3108.

(27) Gan, H.; Fera, D.; Zorn, J.; Shiffeldrim, N.; Tang, M.; Laserson, U. Kim, N.; Schlick, T. RAG: RNA-As-Graphs database-concepts, analysis, and features. Bioinformatics 2004, 20, 1285-1291.

(28) Zahran, M.; Bayrak, C.; Elmetwaly, S.; Schlick, T. RAG-3D: a search tool for RNA 3D substructures. Nucleic Acids Res. 2015, 43, 9474 9488 .

(29) Baba, N.; Elmetwaly, S.; Kim, N.; Schlick, T. Predicting large RNALike topologies by a knowledge-based clustering approach. $\mathrm{J} . \mathrm{Mol}$. Biol 2016, 428, 811-821.

(30) Jain, S.; Saju, S.; Petingi, L.; Schlick, T. An extended dual graph library and partitioning algorithm applicable to pseudoknotted RNA structures. Methods 2019, 162, 74-84.

(31) Jain, S.; Bayrak, C.; Petingi, L.; Schlick, T. Dual Graph Partitioning Highlights a Small Group of Pseudoknot-Containing RNA Submotifs. Genes 2018, 9, 371 .

(32) Schlick, T. Adventures with RNA Graphs. Methods 2018, 143, 16-33.

(33) Jain, S.; Schlick, T. F-RAG: Generating Atomic Models from RNA Graphs using Fragment Assembly. J. Mol. Biol. 2017, 429, $3587-$ 3605 .

(34) Jain, S.; Laederach, A.; Ramos, S.; Schlick, T. A pipeline for computational design of novel RNA-like topologies. Nucleic Acids Res. 2018, 46, 7040-7051.

(35) Zhu, Q.; Schlick, T. A Fiedler Vector Scoring Approach for Novel RNA Motif Selection. J. Phys. Chem. 2021, 125, 1144-1155.

(36) Jain, S.; Tao, Y.; Schlick, T. Inverse Folding with RNA-As-Graphs Produces a Large Pool of Candidate Sequences with Target Topologies. J. Struct. Biol. 2020, 209, 107438.

(37) Huston, N.; Wan, H.; Strine, M.; Tavares, R.; Wilen, C.; Pyle, A. Comprehensive in vivo secondary structure of the SARS-CoV-2 genome reveals novel regulatory motifs and mechanisms. Mol. Cell 2021, 81, 584-598.e5.

(38) Trinity, L.; Lansing, L.; Jabbari, H.; Stege, U. SARS-CoV-2 ribosomal frameshifting pseudoknot: Improved secondary structure prediction and detection of inter-viral structural similarity. bioRxiv, doi: 10.1101/2020.09.15.298604, preprint posted September 2020.

(39) Manfredonia, I.; Nithin, C.; Ponce-Salvatierra, A.; Ghosh, P.; Wirecki, T.; Marinus, T., et al. Genome-wide mapping of SARS-CoV2 RNA structures identifies therapeutically-relevant elements. Nucleic Acids Res. 2020, 48, 12436-12452.

(40) Ahmed, F.; Sharma, M.; Al-Ghamdi, A.; Al-Yami, S.; Al-Salami, A., et al. A Comprehensive Analysis of cis-Acting RNA Elements in the SARS-CoV-2 Genome by a Bioinformatics Approach. Front. Genet. 2020, 11, 1385 .

(41) Andrews, R.; O’Leary, C.; Tompkins, V.; Peterson, J.; Haniff, H.; Williams, C.; Disney, M.; Moss, W. A map of the SARS-CoV-2 RNA structurome. NAR Genom. Bioinform. 2021, 3, lqab043.

(42) Iserman, C.; Roden, C.; Boerneke, M.; Sealfon, R.; McLaughlin, G., et al. Genomic RNA Elements Drive Phase Separation of the SARSCoV-2 Nucleocapsid. Mol. Cell 2020, 80, 1078-1091. 
(43) Kuhlmann, M.; Chattopadhyay, M.; Stupina, V.; Gao, F.; Si- 82 mon, A. An RNA Element That Facilitates Programmed Ribosomal 823 Readthrough in Turnip Crinkle Virus Adopts Multiple Conformations. 824 Virol. J. 2016, 90, 8575-8591.

(44) Moomau, C.; Musalgaonkar, S.; Khan, Y.; Jones, J.; Dinman, J. 826 Structural and Functional Characterization of Programmed Ribosomal 827 Frameshift Signals in West Nile Virus Strains Reveals High Structural Plasticity Among cis-Acting RNA Elements. J. Biol. Chem. 2016, 828 291, 15788-15795.

(45) Houck-Loomis, B.; Durney, M.; Salguero, C., et al. An equilibrium- 83 dependent retroviral mRNA switch regulates translational recoding. Nature 2011, 480, 561-564.

(46) Lu, X.; Bussemaker, H.; Olson, W. DSSR: an integrated software tool for dissecting the spatial structure of RNA. Nucleic Acids Res. 2015, 43, e142-e142.

(47) Abraham, M.; Murtola, T.; Schulz, R., et al. GROMACS: High performance molecular simulations through multi-level parallelism from 837 laptops to supercomputers. SoftwareX 2015, 1-2, 19-25. 838

(48) Schrödinger, LLC, The PyMOL Molecular Graphics System, Ver- ${ }^{839}$ sion 1.8. 2015

(49) Neupane, K.; Zhao, M.; Lyons, A., et al. Structural dynamics of sin- ${ }^{84}$ gle SARS-CoV-2 pseudoknot molecules reveal topologically distinct ${ }^{842}$ conformers. Nat. Commun. 2021, 12, 4749.

(50) Ren, P.; Shang, W.; Yin, W., et al. A multi-targeting drug design strategy for identifying potent anti-SARS-CoV-2 inhibitors. Acta Pharma- ${ }^{845}$ col. Sin. 2021, 0, 1-11.

(51) Park, S.; Kim, Y.; Park, H. Identification of RNA Pseudoknot-Binding ${ }^{847}$ Ligand That Inhibits the -1 Ribosomal Frameshifting of SARS- 848 Coronavirus by Structure-Based Virtual Screening. J. Amer. Chem. ${ }^{849}$ Soc. 2011, 133, 10094-10100.

(52) Neupane, K.; Munshi, S.; Zhao, M.; Ritchie, D.; Ileperuma, S.; Woodside, M. Anti-Frameshifting Ligand Active against SARS Coronavirus-2 Is Resistant to Natural Mutations of the FrameshiftStimulatory Pseudoknot. J. Mol. Biol. 2020, 432, 5843-5847.

(53) Sun, Y.; Abriola, L.; Niederer, R.; Pedersen, S.; Alfajaro, M., et al. Restriction of SARS-CoV-2 replication by targeting programmed -1 ribosomal frameshifting. Proc. Natl. Acad. Sci. USA 2021, 118.

(54) Chen, Y.; Tao, H.; Shen, S.; Miao, Z.; Li, L.; Jia, Y.; Zhang, H.; Bai, X.; Fu, X. A drug screening toolkit based on the -1 ribosomal frameshifting of SARS-CoV-2. Heliyon 2020, 6, e04793.

(55) Wu, M.; D'Souza, V. Alternate RNA Structures. Cold Spring Harb. Perspect. Biol. 2020, 12, a032425.

(56) Feng, S.; Li, H.; Zhao, J.; Pervushin, K.; Lowenhaupt, K.; Schwartz, T.; Dröge, P. Alternate rRNA secondary structures as regulators of translation. Nat. Struct. Mol. Biol. 2011, 18, 169-176.

(57) Mustoe, A.; Brooks, C.; Al-Hashimi, H. Hierarchy of RNA Functional Dynamics. Annu. Rev. Biochem. 2014, 83, 441-466.

(58) Wen, J.; Lancaster, L.; Hodges, C.; Zeri, A.; Yoshimura, S.; Noller, H.; Bustamante, C.; Tinoco, I. Following translation by single ribosomes one codon at a time. Nature 2008, 452, 598-603.

(59) Rangan, R.; Zheludev, I.; Hagey, R.; Pham, E.; Wayment-Steele, H.; Glenn, J.; Das, R. RNA genome conservation and secondary structure in SARS-CoV-2 and SARS-related viruses: a first look. RNA 2020 , 26, 937-959.

(60) Rivas, E.; Eddy, S. A dynamic programming algorithm for RNA structure prediction including pseudoknots. J. Mol. Biol. 1999, 285, 2053 2068.

(61) Hajdin, C.; Bellaousov, S.; Huggins, W.; Leonard, C.; Mathews, D.; Weeks, K. Accurate SHAPE-directed RNA secondary structure modeling, including pseudoknots. Proc. Natl. Acad. Sci. USA 2013, 110, 5498-5503.
(62) Biesiada, M.; Purzycka, K.; Szachniuk, M.; Blazewicz, J.; Adamiak, R. Automated RNA 3D Structure Prediction with RNAComposer. Methods Mol. Biol. 2016, 1490, 199-215.

(63) Xu, X.; Chen, S. Hierarchical Assembly of RNA Three-Dimensional Structures Based on Loop Templates. J. Phys. Chem. B 2018, 122, 5327-5335.

(64) Boniecki, M.; Lach, G.; Dawson, W.; Tomala, K.; Lukasz, P.; Soltysinski, T.; Rother, K.; Bujnicki, J. SimRNA: a coarse-grained method for RNA folding simulations and 3D structure prediction. $\mathrm{Nu}$ cleic Acids Res. 2016, 44, e63-e63.

(65) Krokhotin, A.; Houlihan, K.; Dokholyan, N. iFoldRNA v2: folding RNA with constraints. Bioinformatics 2015, 31, 2891-2893.

(66) Watkins, A.; Rangan, R.; Das, R. FARFAR2: Improved De Novo Rosetta Prediction of Complex Global RNA Folds. Structure 2020, 28, 963-976.e6.

(67) Zgarbová, M.; Otyepka, M.; Šponer, J.; Mládek, A.; Banáš, P.; Cheatham, T.; Jurečka, P. Refinement of the Cornell et al. Nucleic Acids Force Field Based on Reference Quantum Chemical Calculations of Glycosidic Torsion Profiles. J. Chem. 2011, 7, 2886-2902.

(68) Jorgensen, W.; Chandrasekhar, J.; Madura, J.; Impey, R.; Klein, M. Comparison of simple potential functions for simulating liquid water. J. Chem. Phys. 1983, 79, 926-935.

(69) Hess, B.; Bekker, H.; Berendsen, H.; Fraaije, J. LINCS: A linear constraint solver for molecular simulations. J. Comput. Chem. 1997, 18, $1463-1472$

(70) Essmann, U.; Perera, L.; Berkowitz, M.; Darden, T.; Lee, H.; Pedersen, L. A smooth particle mesh Ewald method. J. Chem. Phys. 1995, 103, 8577-8593. 


\section{Supplementary Files}

This is a list of supplementary files associated with this preprint. Click to download.

- covidMDSI.pdf 